\title{
Nonlinear Abel-Type Integral Equation in Modelling Creep Crack Propagation
}

\author{
L. Hakim and S.E. Mikhailov
}

Brunel University West London, UK;

layal.hakim@brunel.ac.uk, sergey.mikhailov@brunel.ac.uk

A non-linear Abel-type equation is obtained in the paper to model creep crack time-dependent propagation in the infinite visco-elastic plane. A finite time when the integral equation solution becomes unbounded is obtained analytically as well as the equation parameters when solution blows up for all times. A modification to the Nyström method is introduced to numerically solve the equation and some computational results are presented.

\subsection{Introduction}

The strength of materials under creep conditions depends not only on the instant value of the load but also on the load duration and generally on the temporal load history. This effect is essential, e.g. for concrete and some plastics under room temperature, and for structural metals under elevated temperatures. The temporal strength condition under constant uniaxial stress, starting at time $t=0$, takes the form

$$
|\sigma|<\sigma^{*}(t) \quad \text { i.e., } \quad \frac{|\sigma|}{\sigma^{*}(t)}<1,
$$

where the dependence of the temporal strength on time has a form shown in Fig. 1.1.

A popular approximation of the temporal strength $\sigma^{*}(t)$ is the power-type function, given by $\sigma^{*}(t)=\sigma^{0} t^{-1 / b}$ that we will call (similar to fatigue) the Basquin diagram, where $\sigma^{0}$ and $b$ are constant material parameters, see e.g. [Ra69, PeMa71].

When the load is not constant but varies with time, different generalizations of relation (1.1), often called accumulation rules, are possible. A novel accumulation rule for life time under variable loading, given in [MiNa09] has the following form, 


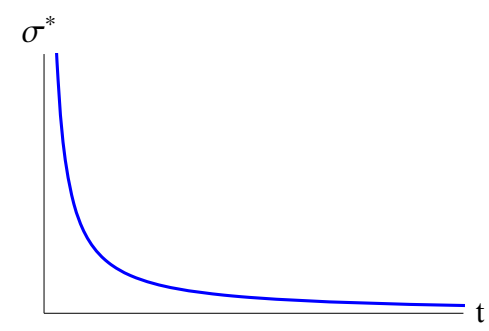

Fig. 1.1. Durability diagram: material strength $\sigma^{*}(t)$ under constant uniaxial stress as function of life time.

$$
\underline{\Lambda}(\sigma ; t):=\left[\frac{d}{d t} \int_{0}^{t}\left|\frac{\sigma(\tau)}{\sigma^{*}(t-\tau)}\right|^{\beta} d \tau\right]^{1 / \beta}<1,
$$

where $\sigma^{*}(t)$ is the durability under constant loading and $\beta \neq 0$ is another material constant.

Particularly for the Basquin diagram $\sigma^{*}(t)=\sigma^{0} t^{-1 / b}$, temporal strength condition (1.2) takes the form,

$$
\begin{aligned}
\underline{\Lambda}(\sigma ; t) & =\left[\frac{d}{d t} \int_{0}^{t}\left|\frac{\sigma(\tau)}{\sigma^{0}}\right|^{\beta}(t-\tau)^{\beta / b} d \tau\right]^{1 / \beta} \\
& =\left[\frac{\beta}{b \sigma_{0}^{\beta}} \int_{0}^{t}|\sigma(\tau)|^{\beta}(t-\tau)^{\frac{\beta}{b}-1} d \tau\right]^{1 / \beta}<1 .
\end{aligned}
$$

As remarked in [MiNa09, MiNa11], for $\beta=b$ the above strength condition is equivalent to the Robinson partial life time linear accumulation rule.

Further generalizations are needed for the multiaxial loading. One of them, that we will further employ, is substituting the maximal principal stress for $\sigma$ in (1.2) and (1.3).

Let us apply temporal strength condition (1.3) to predict crack propagation in a visco-elastic isotropic infinite plane loaded at infinity at time $t=0$ by a traction $q$ constant in space and time, see Fig. 1.2.

Let the crack have a length of $2 a(\tau)$. The stress distribution $\sigma_{22}\left(\tau ; x_{1}\right)$ ahead of the crack in an infinite elastic isotropic (or orthotropic) plane has the following form (e.g. [Sa61]), which remains valid also for the visco-elastic material, see [Ra80],

$$
\sigma_{22}\left(\tau ; x_{1}\right)=\frac{q x_{1}}{\sqrt{x_{1}^{2}-a^{2}(\tau)}} .
$$

Note that due to the problem symmetry, $\sigma_{22}$ is a principal stress component at $x_{2}=0$.

Assuming that the durability of the visco-elastic material is described by the durability condition given in (1.3) with $\sigma=\sigma_{22}\left(\tau, x_{1}\right)$, we substitute there 


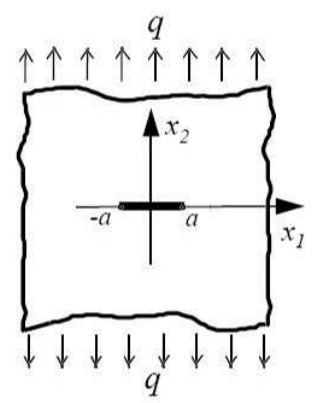

Fig. 1.2. Crack in an infinite plane

stress distribution (1.4). Replacing the inequality with the equality at the tip of the propagating crack, $x_{1}=a(t)$, we arrive at the following nonlinear Volterra integral equation and initial condition for $a(\tau)$,

$$
\begin{aligned}
\int_{0}^{t} \frac{(t-\tau)^{\frac{\beta}{b}-1}}{\left[a^{2}(t)-a^{2}(\tau)\right]^{\beta / 2}} d \tau & =c a^{-\beta}(t), \quad t>0 \\
a(0) & =a_{0}
\end{aligned}
$$

where $b, \beta>0, c=\frac{b}{\beta}\left(\frac{q}{\sigma^{0}}\right)^{-\beta}$ and the initial crack half-length $a_{0}$ are known constants.

Normalising the variables $t \rightarrow t / t_{\infty}$ and $a \rightarrow a / a_{0}$, where $t_{\infty}=\left(\sigma_{0} / q\right)^{b}$ is the life time of the infinite plane without a crack under the same load $q$, simplifies the problem to

$$
\begin{array}{r}
\int_{0}^{t} \frac{(t-\tau)^{\frac{\beta}{b}-1}}{\left[a^{2}(t)-a^{2}(\tau)\right]^{\frac{\beta}{2}}} d \tau=\frac{b}{\beta} a^{-\beta}(t) . \\
a(0)=1,
\end{array}
$$

The function to be sought is $a(t)$, which denotes the normalized length of the crack, and $t$ denotes the normalized time.

\subsection{Integral Equation Characterization}

Equation (1.7) is a non-linear Volterra integral equation and more specifically, since the integrand has a week singularity as $\tau$ approaches $t$, this is an integral equation of the Abel type. The unknown function participates nonlinearly both in the integrand and in the out-of-integral term, and the integrand depends not only on $a(\tau)$ but also on $a(t)$.

For $\beta=b$, equation (1.7) was obtained and solved analytically in [MiNa03a, $\mathrm{MiNa} 03 \mathrm{~b}$. For this case the equation is reduced to a simpler Abel-type integral equation, 


$$
a^{b}(t) \int_{0}^{t} \frac{1}{\left[a^{2}(t)-a^{2}(\tau)\right]^{\frac{b}{2}}} d \tau=1 .
$$

After the change of dependent and independent variables (cf. also [GoVe91]) the equation becomes linear, and the solution can be written as

$$
t(a)=1-\left(\frac{a_{0}}{a}\right)^{2-b} \frac{2 \sin \left(\frac{\pi(b-2)}{2}\right)}{\pi(b-2)}{ }_{2} F_{1}\left(1-\frac{b}{2}, 1-\frac{b}{2}, 2-\frac{b}{2} ; \frac{a_{0}^{2}}{a^{2}}\right)
$$

where ${ }_{2} F_{1}$ is the hypergeometric function.

One can conclude from (1.10) that the solution $a(t)$ of equation (1.9) tends to infinity as $t \rightarrow 1$. That is, the crack propagates through the whole infinite plane in the normalized time $t=1$. Another one conclusion from expression (1.10) is that the solution of (1.9) does exist when $0<b<2$ and blows up when $b \rightarrow 2$. The latter phenomenon also follows from the observation that the integral does not exist in (1.9) for $\beta=2$ for any $t$.

Let us analyse these effects for more general cases of equation (1.7). From mechanical reasonings, we assumed that parameters $b$ and $\beta$ are positive. At any $t$ where the function $a(\tau)$ has a continuous derivative $a^{\prime}(t)$, the integrand in (1.7) tends to

$$
(t-\tau)^{\frac{\beta}{b}-\frac{\beta}{2}-1}\left[2 a(t) a^{\prime}(t)\right]^{-\frac{\beta}{2}}
$$

as $\tau \rightarrow t$. Thus for the integral in (1.7) to exist, we obtain the same condition $0<b<2$ as for equation (1.9).

To find the normalized breaking time $t$ as $a(t)$ tends to infinity, let us multiply both sides of equation (1.7) by $a^{\beta}(t)$ to arrive at the equation

$$
\int_{0}^{t} \frac{(t-\tau)^{\frac{\beta}{b}-1} a^{\beta}(t)}{\left[a^{2}(t)-a^{2}(\tau)\right]^{\frac{\beta}{2}}} d \tau=\frac{b}{\beta}
$$

and consider its limit as $a(t) \rightarrow \infty$. Evidently,

$$
\lim _{a(t) \rightarrow \infty} \frac{a^{\beta}(t)}{\left[a^{2}(t)-a^{2}(\tau)\right]^{\frac{\beta}{2}}}=1 .
$$

Assuming that the limit can be transferred under the integral, the limit of equation (1.11) reduces to

$$
\int_{0}^{t}(t-\tau)^{\frac{\beta}{b}-1} d \tau=\frac{b}{\beta}
$$

Evaluating the integral yields $t^{\frac{\beta}{b}}=1$, which gives $t=1$. Therefore for any $\beta>0$ and $b \in(0,2)$, the normalized breaking time is $t=1$ if the assumption on the limit transfer is correct. 


\subsection{Numerical Algorithms}

No exact or numerical solution of equation (1.7) seems to be available in the literature for cases when $\beta \neq b$. This paper aims to solve numerically equation (1.7) in order to find the normalized crack length, $a$, as the normalized time, $t$, increases, for different values of $\beta$.

There is a wide range of numerical methods for solving Fredholm and Volterra equations. One of the methods is known as the Nyström method and is explained in details e.g. in [At97]. However, the standard Nyström method is not well suited for integral equations with singularities. Therefore, to use the Nyström method for finding approximate solutions to equation (1.7), we modified it as described in the following.

Let us introduce a mesh of $n$ node points, containing equidistant time steps, $t_{i}=i h$, where $h$ is the step size, $i=0,1,2, \ldots, n$. Then, we apply collocations for equation (1.7) in the node points starting from $i=1$,

$$
\int_{0}^{t_{i}} \frac{\left(t_{i}-\tau\right)^{\frac{\beta}{2}-1}}{\left[a^{2}\left(t_{i}\right)-a^{2}(\tau)\right]^{\frac{\beta}{2}}} d \tau=\frac{b}{\beta} a^{-\beta}\left(t_{i}\right), \quad i=1,2, \ldots, n .
$$

To handle the singularity, we split the integral into two parts,

$$
\begin{gathered}
\int_{0}^{t_{i-1}} \frac{\left(t_{i}-\tau\right)^{\frac{\beta}{b}-1}}{\left[a^{2}\left(t_{i}\right)-a^{2}(\tau)\right]^{\frac{\beta}{2}}} d \tau+\int_{t_{i-1}}^{t_{i}} \frac{\left(t_{i}-\tau\right)^{\frac{\beta}{b}-1}}{\left[a^{2}\left(t_{i}\right)-a^{2}(\tau)\right]^{\frac{\beta}{2}}} d \tau=\frac{b}{\beta} a^{-\beta}\left(t_{i}\right) \\
i=1,2, \ldots, n,
\end{gathered}
$$

where the first integral disappears if $i=1$.

First, we use linear interpolation to approximate the square bracket term in the denominator of the second integral,

$$
a^{2}\left(t_{i}\right)-a^{2}(\tau) \approx\left[a^{2}\left(t_{i}\right)-a^{2}\left(t_{i-1}\right)\right] \frac{t_{i}-\tau}{h}, \quad t_{i-1} \leq \tau \leq t_{i}, \quad i=1,2, \ldots, n .
$$

Then substituting this approximation into the second integrand of equation (1.13), applying a quadrature rule to the first integral and denoting $a_{i}=a\left(t_{i}\right)$, we reduce problem (1.7)-(1.8) to the following triangular algebraic system for $a_{j}, j=0, \ldots, n$,

$$
\begin{aligned}
& a_{0}=1, \\
& \sum_{j=0}^{i-1} \frac{\left(t_{i}-t_{j}\right)^{\frac{\beta}{b}-1}}{\left[a_{i}^{2}-a_{j}^{2}\right]^{\frac{\beta}{2}}} w_{i-1, j}+\left(\frac{h}{a_{i}^{2}-a_{i-1}^{2}}\right)^{\frac{\beta}{2}} \int_{t_{i-1}}^{t_{i}}\left(t_{i}-\tau\right)^{\frac{\beta}{b}-1-\frac{\beta}{2}} d \tau \\
& =\frac{b}{\beta} a_{i}^{-\beta}, \quad i=1,2, \ldots, n,
\end{aligned}
$$

where $w_{i-1, j}$ represent the quadrature weights. To make equation (1.16) valid for $i=1$ we set $w_{0, j}=0$. 
Evaluating the integral and simplifying the result, we can rewrite equation (1.16) as

$$
\sum_{j=0}^{i-1} \frac{\left(t_{i}-t_{j}\right)^{\frac{\beta}{b}-1}}{\left[a_{i}^{2}-a_{j}^{2}\right]^{\frac{\beta}{2}}} w_{i-1, j}+\frac{h^{\frac{\beta}{b}}}{\left(\frac{\beta}{b}-\frac{\beta}{2}\right)\left[a_{i}^{2}-a_{i-1}^{2}\right]^{\frac{\beta}{2}}}=\frac{b}{\beta} a_{i}^{-\beta} \quad i=1, \ldots, n .
$$

Since the summation term vanishes in (1.17) for $i=1$, we can find from (1.15) and (1.17) an explicit expression for $a_{1}$ in terms of $h, \beta$ and $b$,

$$
a_{1}=\left(1-\left[\frac{\beta}{b} h^{\frac{\beta}{b}}\left(\frac{\beta}{b}-\frac{\beta}{2}\right)^{-1}\right]^{\frac{2}{\beta}}\right)^{-\frac{1}{2}} .
$$

To find $a_{i}$ for $i \geq 2$ we will use a numerical algorithm. The trapezium quadrature rule was used further in this paper, i.e., the quadrature weights in equation (1.17) have the form

$$
w_{i-1, j}= \begin{cases}\frac{h}{2} & \text { for } j=0 \text { or } j=i-1 \\ h & \text { otherwise }\end{cases}
$$

Substituting the weights and making simplifications, equation (1.17) can be written as

$$
\begin{aligned}
F\left(a_{i}, i\right):= & \frac{h\left(t_{i}-t_{0}\right)^{\frac{\beta}{b}-1}}{2\left[a_{i}^{2}-a_{0}^{2}\right]^{\frac{\beta}{2}}}+h \sum_{j=1}^{i-2} \frac{\left(t_{i}-t_{j}\right)^{\frac{\beta}{b}-1}}{\left[a_{i}^{2}-a_{j}^{2}\right]^{\frac{\beta}{2}}} \\
& +\frac{h^{\frac{\beta}{b}}\left(1+2\left(\frac{\beta}{b}-\frac{\beta}{2}\right)^{-1}\right)}{2\left[a_{i}^{2}-a_{i-1}^{2}\right]^{\frac{\beta}{2}}}-\frac{b}{\beta} a_{i}^{-\beta}=0, \quad i=2,3, \ldots, n .
\end{aligned}
$$

For a fixed $i$, we will solve non-linear equation (1.19) for $a_{i}$ using the Newton method. Thus, each $a_{i}$ will be approximated using the following iterative relation

$$
a_{i, k+1}=a_{i, k}-\frac{F\left(a_{i, k}, i\right)}{F^{\prime}\left(a_{i, k}, i\right)} \quad i=2, \ldots, n .
$$

where $F^{\prime}\left(a_{i}, i\right)$ denotes the derivative of $F\left(a_{i}, i\right)$ with respect to $a_{i}$. Here, $i$ refers to the collocation point in the mesh, while $k$ is the iteration step in the Newton method. Differentiating and simplifying the result yields the following expression for the derivative of $F\left(a_{i}, i\right)$,

$$
\begin{aligned}
F^{\prime}\left(a_{i}, i\right)= & -\frac{h \beta a_{i}\left(t_{i}-t_{0}\right)^{\frac{\beta}{b}-1}}{2\left[a_{i}^{2}-a_{0}^{2}\right]^{\frac{\beta}{2}+1}}-h \sum_{j=1}^{i-2} \frac{\beta a_{i}\left(t_{i}-t_{j}\right)^{\frac{\beta}{b}-1}}{\left[a_{i}^{2}-a_{j}^{2}\right]^{\frac{\beta}{2}+1}} \\
& -\frac{h^{\frac{\beta}{b}} \beta a_{i}\left(1+2\left(\frac{\beta}{b}-\frac{\beta}{2}\right)^{-1}\right)}{2\left[a_{i}^{2}-a_{i-1}^{2}\right]^{\frac{\beta}{2}}}+b a_{i}^{-\beta-1}, \quad i=2, \ldots, n .
\end{aligned}
$$


To avoid the numerical problems related with the infinite growth of $a(t)$ as $t \rightarrow 1$ while computing the iterations in the Newton method solving equation (1.16), we introduced a new variable,

$$
A_{i}=\frac{1}{a_{i}^{2}} .
$$

Thus, by substituting (1.21) in (1.16) and dividing it by $A_{i}^{\beta / 2}$, we arrive at the equation

$$
\begin{aligned}
G\left(A_{i}, i\right) & =\frac{h\left(t_{i}-t_{0}\right)^{\frac{\beta}{b}-1}}{2\left[1-\frac{A_{i}}{A_{0}}\right]^{\beta / 2}}+\frac{h^{\frac{\beta}{2}}\left(1+2\left(\frac{\beta}{b}-\frac{\beta}{2}\right)^{-1}\right)}{2\left[1-\frac{A_{i}}{A_{i-1}}\right]^{\frac{\beta}{2}}}+\sum_{j=1}^{i-2} \frac{h\left(t_{i}-t_{j}\right)^{\frac{\beta}{b}-1}}{\left[1-\frac{A_{i}}{A_{j}}\right]^{\frac{\beta}{2}}} \\
& -\frac{b}{\beta}=0, \quad i=2,3, \ldots, n
\end{aligned}
$$

Calculating the derivative of $G$ with respect to $A_{i}$ gives

$$
\begin{aligned}
G^{\prime}\left(A_{i}, i\right)= & \frac{\beta h\left(t_{i}-t_{0}\right)^{\frac{\beta}{b}-1}}{4 A_{0}\left[1-\frac{A_{i}}{A_{0}}\right]^{\frac{\beta}{2}+1}}+\frac{\beta h^{\frac{\beta}{b}}\left(1+2\left(\frac{\beta}{b}-\frac{\beta}{2}\right)^{-1}\right)}{4 A_{i-1}\left[1-\frac{A_{i}}{A_{i-1}}\right]^{\frac{\beta}{2}+1}} \\
& +\sum_{j=1}^{i-2} \frac{\beta h\left(t_{i}-t_{j}\right)^{\frac{\beta}{b}-1}}{2 A_{j}\left[1-\frac{A_{i}}{A_{j}}\right]^{\frac{\beta}{2}+1}},
\end{aligned}
$$

and we can find values of $A_{i}$ by using the Newton method

$$
A_{i, k+1}=A_{i, k}-\frac{G\left(A_{i, k}, i\right)}{G^{\prime}\left(A_{i, k}, i\right)} \quad \text { where } \quad i=2,3, \ldots, n
$$

and then obtain $a_{i}=1 / \sqrt{A_{i}}$.

This algorithm modification can be associated with solving numerically the equation in the form of equation (1.11) instead of (1.7).

\subsection{Numerical Results}

The described algorithms were implemented using MATLAB as a programming language. As was already discussed, we are interested in the parameter ranges $\beta>0,0<b<2$. The numerical examples given in this paper are for $b=1.5$ and several values of $\beta$.

First, we consider the case $\beta=b$, for which exact solution (1.10) is available, and plot on the same graph, Fig. 1.3, the exact and numerical solutions 


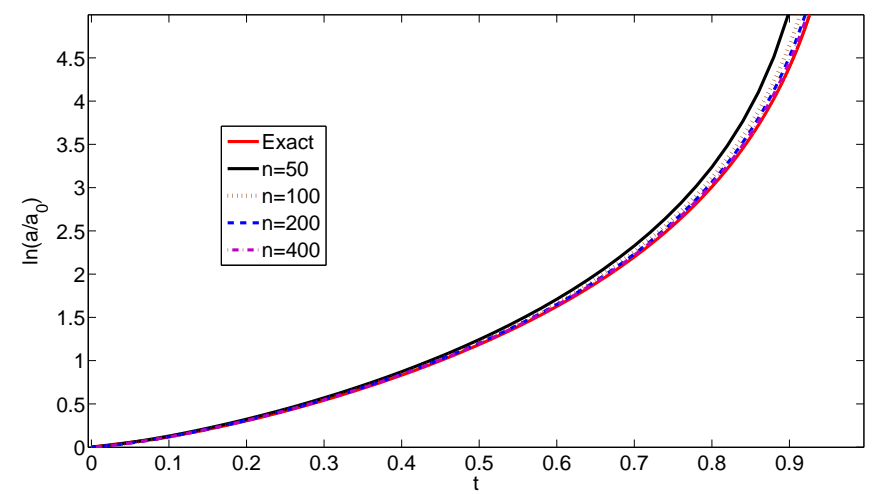

Fig. 1.3. Creep crack length vs. normalized time for different $n, \beta=b=1.5$.

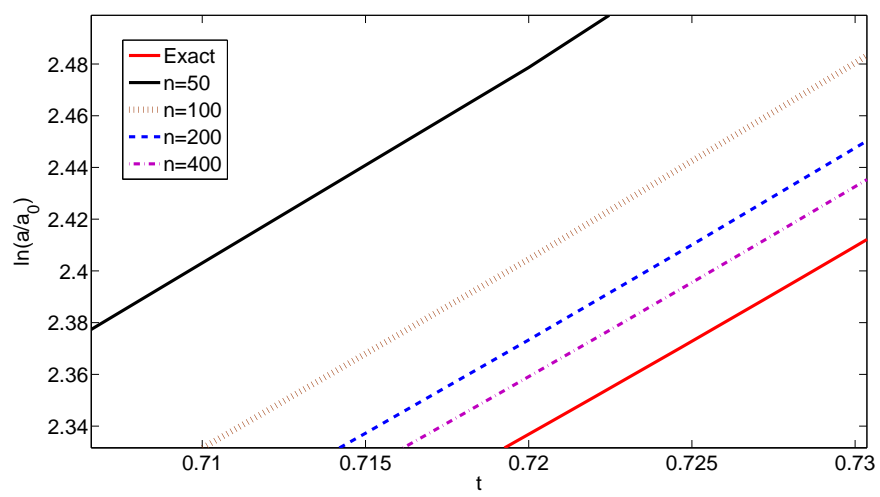

Fig. 1.4. Zoomed in version of the graphs from Fig. 1.3.

for $a$ verses $t$ for different numbers of steps, $n$, on the interval $0<t<1$. The zoomed in part of the plot is given in Fig. 1.4. The graphs show the numerical solution convergence to the exact solution, as $n$ increases.

To quantify the convergence, let us consider the relative error

$$
\epsilon=\frac{\max _{i}\left(\left|A_{i, \text { approx }}-A_{i, \text { exact }}\right|\right)}{\max _{i}\left(\left|A_{i, \text { exact }}\right|\right)}
$$

Note that $\max _{i}\left(\left|A_{i \text {,exact }}\right|\right)=A_{0}=a_{0}^{-2}=1$ in our case. For $b=1.5$, the graph in Fig. 1.5 represents the error verses $n$, where both axes are in logarithmic scale.

One can expect that the convergence at other values of the parameter $\beta$, where no analytical solution is available, will have the same character as for $\beta=b$ and, particularly, for the number of steps, $n=500$, the error in $A$ will not exceed $1 \%$. 


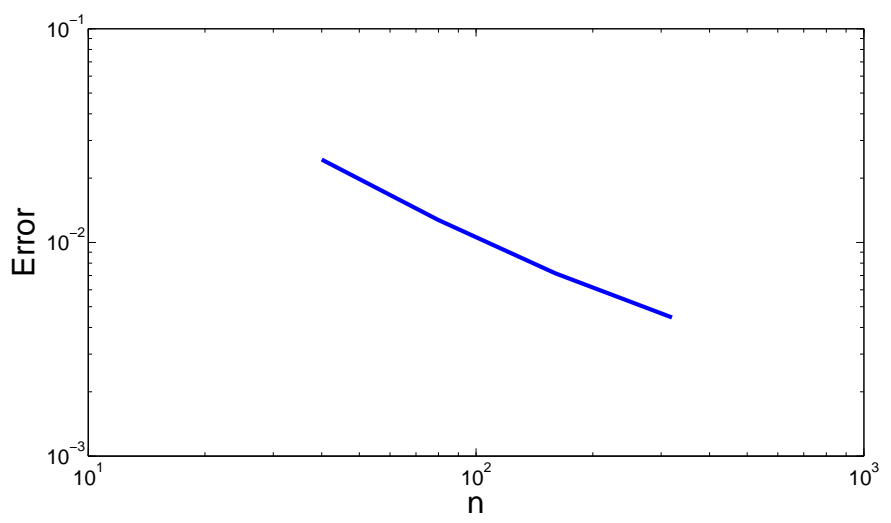

Fig. 1.5. Error for $A$ vs. $n$ for $b=\beta=1.5$

Figs. 1.6, 1.7 present numerical results for the cases $\beta=2 b, \beta=b / 2$, and $\beta=1$ with $n=500$ on the graphs of $\ln \left[a(t) / a_{0}\right]$ verses $t$, and the graphs of $A(t) / A_{0}$ verses $t$.

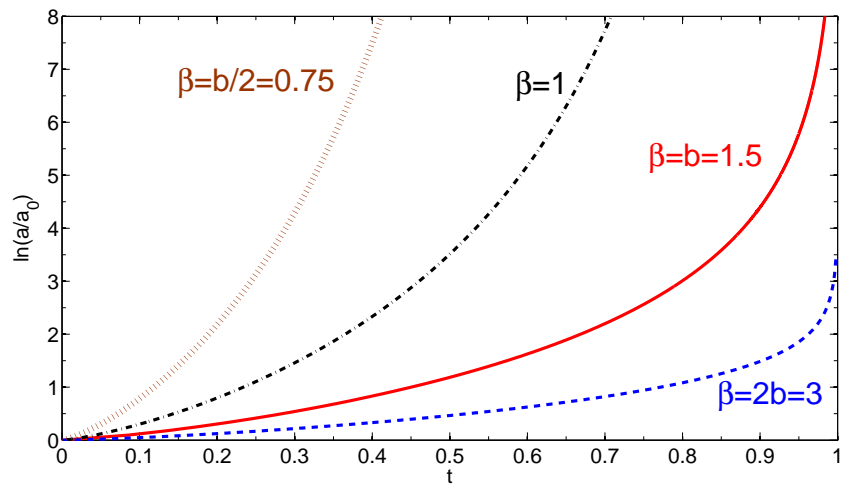

Fig. 1.6. Creep crack length vs. normalized time for $b=1.5$

Graphs representing $A(t)$ are particularly useful for analysing the breaking time; this is when the curve crosses the time axes, since the crack length $a=1 / A^{2}$ tends to infinity when $A$ tends to zero. From the graph in Fig. 1.7, we can see that when $\beta=2 b$ the normalized breaking time is $t=1$. For other values of $\beta$, it is not completely clear from the graphs but we come to the same conclusions under the assumption made in Section 1.2. 


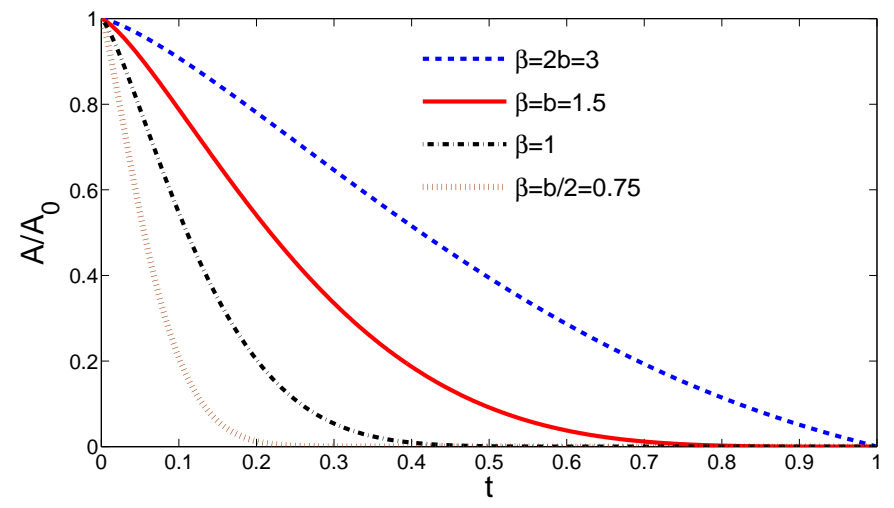

Fig. 1.7. $A$ vs. normalized time for $b=1.5$

\subsection{Conclusion}

A non-linear Abel-type equation, which kernel depends on the unknown function at several values of the variable, was obtained in the paper to model creep crack propagation in the infinite visco-elastic plane.

A modification to the Nyström method was introduced to numerically solve the equation. The numerical results obtained for the special case $\beta=$ $b$, and the analysis of the error with respect to the mesh size, demonstrate convergence of the modified Nyström method and indicate that this scheme can be applied to solve the integral equation with other values of the parameter $\beta$ and also more general nonlinear Abel-type equations.

From the results on the creep crack growth presented in the paper, one can conclude that an increase of the parameter $\beta$ decreases the crack growth rate. The theoretical analysis of the integral equation shows that its solution blows up at $b \geq 2$ for any $\beta>0$. On the other hand, for $0<b<2$ and any $\beta>0$, the normalized breaking time for an infinite plane with a crack is $t=1$.

Under some fatigue models, the analysis of fatigue crack propagation in an elastic material can be similarly reduced to equation (1.7), where the time, $t$, should be however replaced with the number of load cycles, $n$, cf. [MiNa03a]. One can use this similarity to make corresponding conclusions about the fatigue crack propagation as well.

\section{References}

[At97] Atkinson, K.E.: The Numerical Solution of Integral Equations of the Second Kind, Cambridge University Press, New York-Melbourne (1997).

[GoVe91] Gorenflo, R., Vessella, S.: Abel Integral Equations Analysis and Aplications. Springer Verlag, Berlin-New York (1991). 
[MiNa03a] Mikhailov S.E., Namestnikova I.V.: Local and Non-Local Approaches to fatigue Crack Initiation and Propagation. In: Movchan A.B. (ed) IUTAM Symposium on Asymptotics, Singularities and Homogenisation in Problems of Mechanics. Kluwer, The Netherlands, 285-294 (2003).

[MiNa03b] Mikhailov, S.E., Namestnikova, I.V.: Local and Non-Local Approaches to Creep Crack Initiation and Propagation. In: Proceedings of the 9th International Conference on the Mechanical Behaviour of Materials. Geneva, Switzerland (2003).

[MiNa09] Mikhailov, S.E., Namestnikova, I.V.: About accumulation rules for lifetime prediction under variable loading. In: Proceedings of the 12th International Conference on Fracture, Ottawa, Canada. Natural Resources Canada \& National Research Council of Canada (2009).

[MiNa11] Mikhailov, S. E., Namestnikova I. V.: History-sensitive accumulation rules for life-time prediction under variable loading. Archive of Applied Mechanics, DOI: 10.1007/s00419-011-0511-6, (2011).

[PeMa71] Penny, R., Marriott, D.: Design for Creep. McGraw-Hill, London (1971).

[Ra69] Rabotnov, Y.N.: Creep Problems in Structural Members. North-Holland Publ., Amsterdam-London (1969).

[Ra80] Rabotnov, Yu. N.: Elements of Hereditary Solid Mechanics, Mir Publishers, Moscow (1980).

[Sa61] Savin G.N.: Stress concentration around holes, Oxford, Pergamon Press (1961). 